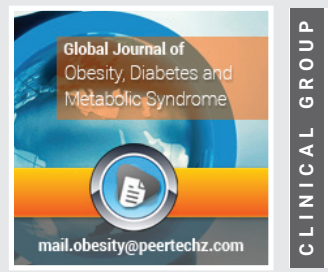

\title{
Management of Diabetic Foot Ulcer- A case study
}

\author{
Gouri Sakre $^{1 *}$ and Suresh Kishanrao² \\ ${ }^{1}$ BHMS, MPH, Karnataka State Rural Development \&Panchayat Raj University (KSRDPRU) Gadag, \\ Bengaluru, Karnataka, India \\ ${ }^{2} \mathrm{MD}$, DIH, FIAP, FIPHA, FISCD, Public Health Consultant and Visiting Professor- MPH. Karnataka State \\ Rural Development \&Panchayat Raj University (KSRDPRU) Gadag, Bengaluru, Karnataka, India
}

Received: 10 April, 2021

Accepted: 26 April, 2021

Published: 27 April, 2021

*Corresponding author: Dr. Gouri Sakre, BHMS, MPH, Karnataka State Rural Development\& Panchayat Raj University (KSRDPRU) Gadag, Bengaluru, Karnataka, India, E-mail: gourisakre.1993@gmail.com

https://www.peertechzpublications.com

\section{Check for updates}

\begin{abstract}
Diabetic Foot Ulcers (DFU) is a common complication among neglected management or long-term diabetes in developing countries like India for want of requisite services. DFU is estimated to be affecting approximately $15 \%$ of diabetic patients during their lifetime. Other complications of diabetes include Diabetic retinopathy and glaucoma affecting eyes, mouth problems like gingivitis and periodontitis, Alzheimer's disease affecting brain, diabetic retinopathy affecting hands and legs, Erectile Dysfunction (ED) among men, kidney failures. From the public health point of view, Diabetic Foot Ulceration is important as it is preventable. Globally recommended measures include good control of blood sugar, $\mathrm{HbA1c}$, haemoglobin, blood pressure, and lipid levels that help to reduce amputations up to $80 \%$. Diabetic foot ulcers are common on pressure points like heels, toes, and plantar surfaces.
\end{abstract}

We report a rare case here due to 2 reasons 1 . An unusual site 2. DFU development within a short duration of 2 years after diagnosis. A young lady in her late 30 's developed foot ulcer over left lower malleolus because of the pressure point due to the professional practice (tailor) of squatting on the floor to work within 2 years of diagnosis of diabetes. Started as bilateral pedal oedema, that progressed to inflammation and induration of tissues over left malleolus. In a week's time it increased and started oozing non-purulent exudate. An initial antibiotic therapy gave some relief but recurred with purulent discharge after 2 weeks of stopping antibiotic. A surgical debridement and approximation of the edges of the ulcer was done to facilitate the growth of the epithelium across the ulcer bed. The wound took over a week's antibiotic therapy after debridement and over 3 months of insulin therapy and regular wound dressing to heal fully.

\section{Background}

India contributes approximately 42 million cases out of a global burden of 463 million cases and it is ranked first in the list of the ten nations most affected with diabetes. Diabetic foot diseases including Diabetic foot ulceration (DFU) are common complications among poorly managed or long duration diabetes patients in India. Diabetic foot affects approximately $15 \%$ of diabetic patients during their lifetime [1]. Among diabetes patients peripheral nerve damages often occur leading to loss of pain sensation, and damages to skin by pressure or injury leading the ulceration without their knowledge. Foot ulceration and its poor management leads to 85 percent of diabetesrelated amputations of lower limbs. Individual characteristics like overweight, alcohol and tobacco consumption accelerate the process of foot ulcers development. Vascular disease can complicate a foot ulcer, reducing the body's ability to heal and increasing the risk for an infection. Consistent high blood glucose levels reduce the body's ability to fight off a potential infection and delays the healing process. Several social and cultural practices like barefoot walking, squatting on the floor for long hours by certain professionals like tailors, priests, house maids etc. add to risk factors. Inadequate facilities for diabetes screening, blood sugar monitoring and management and foot care on one hand and use of the available facilities for diabetes care and education, and poor socioeconomic conditions are the main causes for foot ulcers in developing countries including India.

\section{Case presentation}

A young woman of thirty-eight-year-old, tailor by profession, reported with an oozing swelling over left malleolus (ankle) in January 2020. She is used to sit on the ground, crossed legs on floor for cutting the clothes for stitching. The posture led to lot of pressure on the left lateral (malleolus) at the knob on outside of the ankle. She was a known type 2 diabetic since April 2019 on oral anti-diabetic's therapy and 
diet control. However, detailed history indicated that she was on irregular treatment and unrestricted diet plan. The first sign of the complication was bilateral pedal oedema. Soon the skin and tissue over left lateral malleolus, started swelling (painless induration) and oozing of non-purulent exudates. The condition went on worsening over a period of one month (Feb 2020 to March 2020), leading to purulent discharge, induration, edema around the pustule with no pain. Initially a family doctor suggested limb elevation and rest between the works, to minimize the pressure on the affected limb. Then a physician in the first week of February 2020, put her (Tab. Augmentin 625 (Amoxicillin and Clavulanic acid combination) twice daily $\times 3$ days and Tab. Zerodol SP-(a combination of Aceclofenac, Paracetamol and Serrati peptidase.) twice daily×3days an antibiotics and anti-inflammatory, respectively. There was an improvement in the swelling, exudates stopped oozing for ten days after treatment. She was put on oral antidiabetic Tab. Glycomet (Metformin) SR 500mg twice daily only despite her RBS level being 250-300mg/dL.

\section{Surgery}

After fifteen days same complaints recurred, and she was taken to Private surgery in Hubli. There a general surgeon examined the site and advised debridement of the affected area. On $17^{\text {th }}$ March, the debridement and epithelial edge advancement of the wound at the left lateral malleolus (Figure 1) was done.

1. Preoperative investigations includedComplete blood count: Hb\% - 11.5, WBC - 7500, Platelets - 1,38,000.

2. HbA1c - 9.2\%,3. HbsAg - Negative, 4. HIV - Negative.

\section{Post- operative treatment included Tab}

Augmentin 625mg× 5days, Tab. Zerodol $\times$ 5days, strict limb elevation, alternate day dressing. The treatment was based on empirical prevalence of antibiotic sensitivity in the city based on the surgeon's experience, no culture and sensitivity facility were used. The next day after surgery there was lot of soakage of the dressings at the site. After 5 days of surgery $\left(23^{\text {rd }}\right.$ March 2020) the surgeon inspected the wound and took

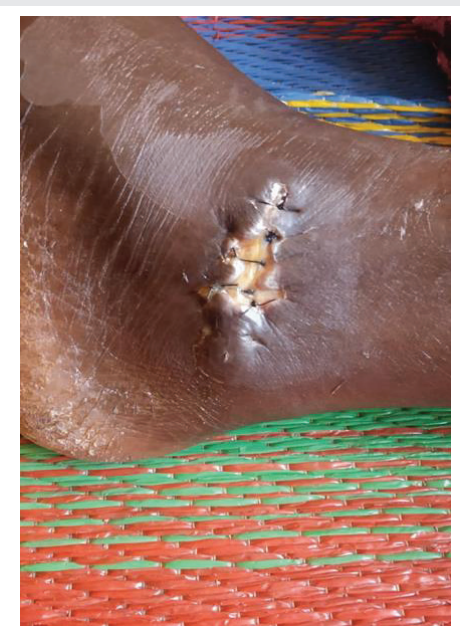

Figure 1: Debrided Ulcer on Left Malleolus. stock of her daily BGL (which ranged between 250 to $350 \mathrm{mg} /$ dl since surgery) and advised to consult a physician as the wound condition was worsening. The Physician put her on regular insulin Inj. Lantus (Insulin glargine), and Tab. Linid (Linezolid) 600mg BD for 5days, as the blood sugar level fluctuated between 140 to $340 \mathrm{mg} / \mathrm{dl}$ over the last one month. After starting (20 units) Insulin twice daily (duly adjusting the dose based on BGL) the wound started healing slowly.

\section{Follow-up}

The next follow up with the surgeon was disrupted due to nation-wide lockdown for controlling the spread of Covid 19 Pandemic. Out of 5 sutures the first and third suture were removed at home on $6^{\text {th }}$ of April. The peeling of skin, indurations over the site of surgery and blackish discoloration of the foot demanded continuation of insulin. The Lantus injection was continued for about three months adjusting the insulin dose according to the sliding scale $101-150 \mathrm{mg} / \mathrm{dl}=10$ units, 151200mg/dl=12units, $201-250=14$ units, $251-300=16$ units, and $301-350=18$ units.

\section{Details of insulin therapy}

Insulin was started on $25^{\text {th }}$ March 2020 FBS $193 \mathrm{mg} /$ $\mathrm{dl}=12$ units, $27 / 03 / 2020 \mathrm{FBS}=203,14$ units, 10/4/2020 FBS=160, 12units, from $11 / 04 / 2020$ to $13 / 4 / 2020$ FBS was between $150-$ 200, 12 units. There was break on 09/05/2020 and 10/05/21. On 10/05/2020 as HbA1c was 11.1 and FBS - 138, PPBS - 340. Inj. insulin was resumed on 11/05/2020 and tapered till 22/05/2020 by reducing two units each day. Other treatment included oral anti-diabetic drugs Tab. Glimepiride $3 \mathrm{mg}$ before each meal and Tab. Metformin 1000mg after each meal, and daily dressing for 3 months to hasten the wound recovery. Wound started healing by May 2020, after 3 months it healed fully (Figure 2 ).

\section{Discussion}

The lifetime risk of a person with diabetes having a foot ulcer is around $25 \%$ and is the commonest reason for hospitalization of diabetic patients (about 30\%) and absorbs about $20 \%$ of the total health-care costs, more than all other diabetic complications. The prevalence of foot ulcers in all diabetics attending a center managing diabetic foot in India was $3 \%$ in 2015 . Foot ulcers among outpatient and inpatient diabetics attending hospitals across rural India were found to be $10.4 \%$ Over all $15 \%$ of diabetic patients in India are affected with DFU during their lifetime [1,2]. Approximately, $85 \%$ of non-traumatic lower limb amputations are seen in patients with prior history of diabetic foot ulcer [1] Age of the patients, duration of diabetes, alcohol and tobacco consumption, inadequate oral hypoglycemic /insulin treatment, poor foot care access and rural location and accessing local remedies are important risk factors that worsen the diabetes and lead to complications. Several social and cultural practices like barefoot walking, squatting on the floor for long hours by certain professionals like tailors, priests, house maids etc. inadequate facilities especially in rural remote areas and poor utilization of the available facilities for diabetes care and education, and poor

Citation: Gouri Sakre, K Suresh (2021) Management of Diabetic Foot Ulcer- A case study. Glob J Obes Diabetes Metab Syndr 8(1): 001-005. DOI: https://dx.doi.org/10.17352/2455-8583.000049 


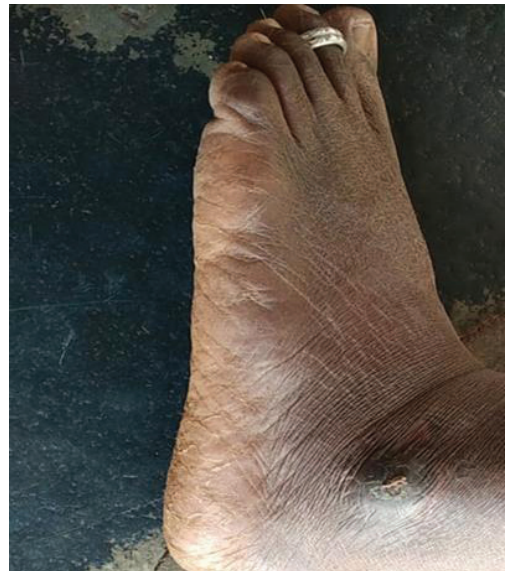

Figure 2: Fully healed Ulcer after 3 months.

socioeconomic conditions prohibiting monitoring are the main causes for foot ulcers.

It is estimated that approximately 45,000 legs are amputated every year in India and the vast majority (75\%) of these result from an infected neuropathic foot. More than half of all foot ulcers become infected, requiring hospitalization, while $20 \%$ of infections result in amputation. People with a history of diabetic foot ulcer have a $40 \%$ greater 10 -year death rate than people with diabetes alone [1].

In a recent study to evaluate foot-care practices by physicians throughout India who had participated in the Diabetic Foot Education Program out of a total of 377 doctors including 261 doctors who belonged to independent diabetic foot clinics, $44.4 \%$ reported managing fewer than five diabetic foot patients per week and $42.8 \%$ reported managing 5-10 patients per week. Whereas 58\% reported performing comprehensive clinical examinations and providing optimal preventive and therapeutic care, only $25.7 \%$ reported performing only callus removal and changing dressings. The most common comorbidities were neuropathy and peripheral vascular diseases. The study reaffirms the need to promote diabetic foot awareness and implement foot-care strategies to prevent diabetic foot and effectively manage this condition [3].

An observational study in North India reported $14 \%(n=97)$ diabetic foot ulcers (DFUs) among 678 diabetic patients. Of the remaining 581 patients with no foot ulcers, $42.16 \%$ belonged to rural areas as against $70.1 \%$ among the DFUs cases were from rural areas indicating the poor diabetes management and lack of foot care services. Important risk factors identified for DFUs in that study were age $>50$ years, 4 to 8 years of diabetes $(\mathrm{OR}=$ $2.47)$ and $>8$ years $(\mathrm{OR}=3.03$,$) , rural location (\mathrm{OR}=0.44$,$) , Oral$ hypoglycemic treatment $(\mathrm{OR}=2.90)$ insulin treatment $(\mathrm{OR}=$ 9.58$,$) , and tobacco use (\mathrm{OR}=0.57)$ by a multivariate logistic regression analysis [4].

In another recent diabetic foot infection prospective study, in PGI Chandigarh $(\mathrm{n}=65)$ male predilection $(83.08 \%)$ and a mean duration of diabetes mellitus was $12.03 \pm 6.96$ years were followed up for 3 months. Only 1 case $(1.54 \%)$ was in the age group of our case. Ulcer (92.31\%) and discharge (72.31\%) were the most common presenting complaints. Monomicrobial growth was present in 36 patients $(55.38 \%)$. The most common isolates were Escherichia coli and Staphylococcus aureus (28.57\% each). This study indicated that the predominance of monomicrobial growth and Gram-negative organisms in diabetic foot patients is common. The mean baseline area of ulcer was $14.85 \mathrm{~cm} \pm 23.12 \mathrm{~cm}$, that reduced to mean ulcer area of $11.75 \mathrm{~cm} \pm 22.68 \mathrm{~cm}$ after 1 month, $8.44 \mathrm{~cm} \pm 22.05 \mathrm{~cm}$ after 2 months and after 3 months mean ulcer areas reduced to was $6.38 \mathrm{~cm} \pm 21.19 \mathrm{~cm}$. Percentage of ulcer healing at 1 month was $20.88 \%$, improving to $43.16 \%$ by 2 months and $57.04 \%$ by 3 months [5].

In another prospective study from multiple diabetic clinics in Hyderabad, India, from January 2016 to August 2018 indicated that the age of the patients with ulcers was in the range of 50 to 70 years in contrast our case. In that study $61 \%$ of the patients had neuropathic ulcer, $20 \%$ ischemic ulcer and $19 \%$ had neuroischemic ulcer. $69 \%$ of these patients had osteomyelitis. $52 \%$ had ulcer on the right foot and $48 \%$ on the left. $15 \%$ had dry gangrene with ulcer whereas $85 \%$ had infected ulcers. $60 \%$ of the ulcers were only on the plantar aspect and $24 \%$ had ulcers all around the toe. None of the ulcers were in the site of our case making it unique with an association with the need of her profession.

Based on 52 years' experience of second author, authors believe that the duration and severity of diabetes, management of bold sugar over year, diabetic food care services availability and utilization coupled with the age and occupation of the patients that determine the pressure points may determine the site of DFU, sometimes unusual sites as in this case. Based on the site of the DFU we consider it to be Neuropathic ulcers in areas overlying a bony surface of malleolus. The commonly reported DFU in India include $60 \%$ on the plantar aspect and $24 \%$ around the toes. Probably this is the first reported case of DFU on the malleolus in India. Mean duration of diabetes mellitus was unusually short in our case in contradiction to other studies in India $\{4-8$ years [1] and $12.03 \pm 6.96$ years [2]\}. The healing time of 3 months in this case was thought

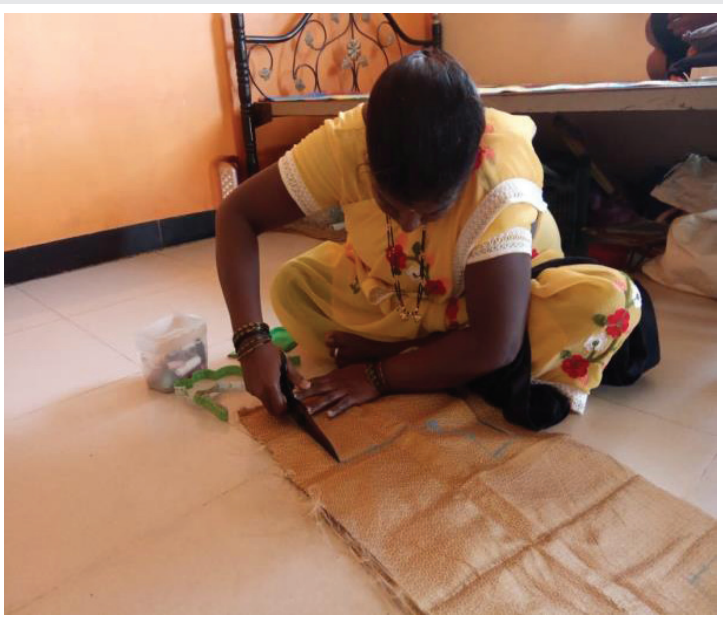

Figure 3: Sitting posture, when bent forward for cutting the body pressure falls on left malleolus

Citation: Gouri Sakre, K Suresh (2021) Management of Diabetic Foot Ulcer- A case study. Glob J Obes Diabetes Metab Syndr 8(1): $001-005$. 
long, but the evidence in India indicates that even by 3 months healing could be less than $60 \%$ [2]. The significant association of severity of DFI with the rate of ulcer healing suggests that our case was diagnosed late [2]. Our case's uniqueness in terms of age, site of the DFU, the duration of diabetes, inadequate management alert us about the probability of other complications like diabetic retinopathy occurring much faster, we need to keep monitoring (Figure 3 ).

\section{International guidelines for management of diabetes foot ulcer and its prevention}

The principle aim of DFU management consist of preventing occurrence of ulcer with better blood sugar maintenance and foot care, addressing risk factors. When it does occur, they should be treated at an early stage to allow prompt healing. The essential processes consist of 1) treating underlying disease processes by a) achieving optimal consistent diabetic control b) minimizing the physical cause of the trauma- 2. Optimal Local wound Care through tissue debridement, inflammation, and infection control. 3. Moisture balance through optimal dressing selection and healthy cut edges proximation 4) Pressure offloading from the site [6].

\section{The international guidelines consist of six evidence- based areas}

1. Prevention of foot ulcers in persons with diabetes: Identifying the at-risk foot, regularly inspecting, and examining the at-risk foot, educating the patient, family, and healthcare professionals, ensuring routine wearing of appropriate footwear and treating risk factors for ulceration are the key areas to concentrate.

2. Offloading foot ulcers in persons with diabetes: Offloading is a cornerstone in treatment of ulcers that are caused by increased biomechanical stress. When DFU is infected or ischemic offloading is still important.

3. Diagnosis, prognosis, and management of peripheral artery disease in DFU and diabetes: In patients with either an ankle pressure $<50 \mathrm{~mm} \mathrm{Hg}$ vascular imaging I to be done and when findings suggest it is appropriate, revascularization is to be taken up

4. Diagnosis and treatment of foot infection in persons with diabetes: Cleansing debridement of all necrotic tissue and surrounding callus and start empiric oral antibiotic therapy targeted at Staphylococcus aureus and streptococci is the recommended approach.

5. Interventions to enhance healing of foot ulcers in persons with diabetes: Optimizing glycemic control, if necessary, with insulin and treating oedema or malnutrition, if present. Regular inspection of the ulcer, debriding the ulcer and using dressings to control excess exudation and maintaining moist environment. The feet, should not be soaked as that may induce skin maceration and apply negative pressure to help heal post-operative wounds.
6. Classification of diabetic foot ulcers: Classification of the ulcers using the IWGDF/ISDA classification criteria helps in better management.

\section{Diabetic Foot management guidelines in India}

There is a lack of a good evidence-based standard guideline on Diabetic foot Care in India. Currently, diabetic feet are treated by individual practitioners. Physicians, General surgeons, orthopedic surgeons, primary care physicians, endocrinologists, and podiatrists.

Sadly, their roles, responsibilities and the protocols are not clearly defined in the public domain.Diabetes foot management in India is based on comprehensive patient and wound assessment. It includes arterial inflow and infection control, the degrees of sensory neuropathy and deformity evaluation, and reduction of trauma (footwear) and pressure (offloading) [1].

Foot care advise includes Inspection of feet daily for cracks, blisters, infections, and injuries. It advises use of a mirror or a magnifying glass to see the sole better or seek help someone else to do it Cleanse feet daily as you bathe or shower, using warm water and mild soap. It also advises to Moisturize dry skin by using oil, lotion or cream and seek doctors help if it causes redness or irritation. Clipping toenails straight across with a nail cutter and not to use a scissor. It also advises wearing something on your feet (socks, slippers, shoes) to protect from injury always even in house. Treating minor breaks in the skin promptly and seeing a doctor to check your feet if there is any redness, swelling or induration [7].

\section{Way forward for india}

The present care plan in India has three basic sections: assessment, general management, and antibiotic therapy. India is far away from the ideal situation as recommended by IWGDF and need to promote diabetic foot awareness and implement foot-care strategies at least each district hospitals. It is important establish podiatric medical care at district level to start with and promote use of the services as early as possible. The Government and the professional bodies should empower the primary care doctors and the non-specialist practitioners with the skills to assess and refer to the multidisciplinary footcare team as early as possible.

\section{Take home messages}

$>$ Consistent good control of blood sugar and other risk factors of DFU

$>$ Once a diabetic foot ulcer is noticed, seek podiatric medical care immediately.

> Treat Foot ulcers as soon as possible to reduce the risk of infection and amputation.

\section{References}

1. The Diabetic foot Prevention and management in India, Ministry of Health \& Family Welfare, Government of India. Link: https://bit.ly/3aAdBt5 
2. Shahi KS, Kumar A, Kumar S, Singh SK, Gupta SK, et al. (2012) Prevalence of Diabetic Foot Ulcer and Associated Risk Factors in Diabetic Patients from North India. Journal of Diabetic Foot Complications 4: 83-91. Link: https://bit.ly/32PYIDW

3. Das A, Pendsey S, Abhyankar M, Malabade R (2020) Management of Diabetic Foot in an Indian Clinical Setup: An Opinion Survey. Cureus 12: e8636. Link: https://bit.ly/3vnq2QH

4. Seth A, Attri AK, Kataria H, Kochhar S, Seth SA, et al. (2019) Clinical profile and outcome in patients of diabetic foot infection. Int J Appl Basic Med Res 9: 14 19. Link: https://bit.ly/3sPtGkY
5. Pilla EDP, Desai R, okiwar RP (2019) A multi centric study of diabetic foot ulcercauses and complications. ISJ 6. Link: https://bit.ly/32R5jJ3

6. The International Working Group on the Diabetic Foot Guidance (IWGDF Guidance). Link: https://bit.ly/3dRHjMg

7. Training Module for Medical Officers for Prevention, Control and Population Level Screening of Hypertension, Diabetes and Common Cancer. Link: https://bit.ly/32MksLs

\section{Discover a bigger Impact and Visibility of your article publication with} Peertechz Publications

\section{Highlights}

* Signatory publisher of ORCID

* Signatory Publisher of DORA (San Francisco Declaration on Research Assessment)

* Articles archived in worlds' renowned service providers such as Portico, CNKI, AGRIS, TDNet, Base (Bielefeld University Library), CrossRef, Scilit, J-Gate etc.

* Journals indexed in ICMJE, SHERPA/ROMEO, Google Scholar etc.

* OAI-PMH (Open Archives Initiative Protocol for Metadata Harvesting)

* Dedicated Editorial Board for every journal

* Accurate and rapid peer-review process

* Increased citations of published articles through promotions

* Reduced timeline for article publication

Submit your articles and experience a new surge in publication services (https://www.peertechz.com/submission).

Peertechz journals wishes everlasting success in your every endeavours.

Copyright: @ 2021 Sakre G, et al. This is an open-access article distributed under the terms of the Creative Commons Attribution License, which permits unrestricted use distribution, and reproduction in any medium, provided the original author and source are credited. 